\title{
PARTICLE CANDIDATES FOR DARK MATTER
}

\author{
John Ellis \\ Theoretical Physics Division, CERN \\ CH - 1211 Geneva 23
}

\begin{abstract}
Some particle candidates for dark matter are reviewed in the light of recent experimental and theoretical developments. Models for massive neutrinos are discussed in the light of the recent atmospheric-neutrino data, and used to motivate comments on the plausibility of different solutions to the solar neutrino problem. Arguments are given that the lightest supersymmetric particle should be a neutralino $\chi$, and accelerator and astrophysical constraints used to suggest that $50 \mathrm{GeV} \lesssim m_{\chi} \lesssim 600 \mathrm{GeV}$. Minimizing the fine tuning of the gauge hierarchy favours $\Omega_{\chi} h^{2} \sim 0.1$. The possibility of superheavy relic particles is mentioned, and candidates from string and $M$ theory are reviewed. Finally, the possibility of non-zero vacuum energy is discussed: its calculation is a great opportunity for a quantum theory of gravity, and the possibility that it is time dependent should not be forgotten.
\end{abstract}

Invited talk presented at the Nobel Symposium

Haga Slott, Sweden, August 1998 


\section{Introduction}

There is a wide range of possible masses for a candidate dark matter particle [1]. If it was once in thermal equilibrium, its number density $n$ is almost independent of its mass, as long as the latter is $\ll 1 \mathrm{MeV}$. Thus, for neutrinos with masses in the range discussed in the next section, $n_{\nu} \sim$ constant and hence $\rho_{\nu}=m_{\nu} n_{\nu} \propto m_{\nu}$, leading to

$$
\Omega_{\nu} h^{2} \equiv\left(\frac{\rho_{\nu}}{\rho_{c}} h^{2}\right) \simeq \sum_{i}\left(\frac{m_{\nu_{i}}}{98 \mathrm{eV}}\right),
$$

where $h$ is the present Hubble expansion rate in units of $100 \mathrm{kms}^{-1} \mathrm{Mpc}^{-1}$ and $\rho_{c}$ is the critical density. There is a region of masses for neutrinos, or similar particles, between $0(100) \mathrm{eV}$ and $0(3) \mathrm{GeV}$ where $\Omega_{\nu}>1$. Above this range, the cosmological density $\rho_{X}$ of a particle $X$ may be sufficiently suppressed by mutual annihilation:

$$
\rho_{X}=m_{X} n_{X}: n_{X} \propto \frac{1}{\sigma_{\text {ann }}(X X \rightarrow \ldots)},
$$

that $\Omega_{X} \lesssim 1$. In the case of neutrinos or similar particles, annihilation is particularly efficient when $m_{\nu} \sim m_{Z / 2}$, leading to a local minimum in the relic density with $\Omega_{\nu} \ll 1$. Above this mass, the relic density in general rises again, depending on the behaviour of the annihilation cross-section. Thus one has three regions where the relic density of a neutrino or similar particle may be of cosmological interest: $\Omega_{\nu} \sim 1$ :

$$
m_{\nu} \sim 30 \mathrm{eV}, \quad \sim \text { few } \mathrm{GeV}, \quad \text { or } \sim 100 \mathrm{GeV} .
$$

The first of these possibilities corresponds to hot dark matter, and is of active interest for neutrinos as discussed in Section 2, whilst the other two correspond to cold dark matter, and are of interest for supersymmetric particles, as discussed in Section 3. The middle region has been essentially excluded by LEP, as we discuss in more detail later, whilst the third may be in the realm of the LHC.

It is of interest for our subsequent discussion to review in more detail [2] the upper limit (3) on the mass of a cold dark matter particle. One may write

$$
\Omega_{X}=\frac{\rho_{X}}{\rho_{c}} \simeq \frac{m_{X} n_{X}}{2 \times 10^{4} h^{2} T_{0}^{2}}
$$

where $T_{0} \simeq 2.73 \mathrm{~K}$ is the present effective temperature of the cosmic microwave background radiation. To a good approximation, the comoving number density has remained essentially constant since the freeze-out temperature $T_{f}$ at which annihilation terminated:

$$
\frac{n_{X}}{T_{0}^{3}} \simeq \frac{n_{X}\left(T_{f}\right)}{T_{f}^{3}}: n_{X}\left(T_{f}\right)\left\langle\sigma_{\mathrm{ann}}(X X) v_{X}\right\rangle=\frac{\dot{a}}{a} \simeq \frac{T_{f}^{2}}{m_{P}},
$$

where $a$ is the cosmological scale factor and $m_{P} \simeq 1.2 \times 10^{19} \mathrm{GeV}$ is the Planck mass. For relic particles of interest, one typically finds that $m_{X} / T_{f} \sim 20$ to 30 and hence

$$
\Omega_{X} h^{2} \simeq \frac{10^{-3}}{\left\langle\sigma_{\mathrm{ann}}(X X) v_{X}\right\rangle} \times\left(\frac{1}{T_{0} m_{P}}\right) \simeq \frac{10^{-3}}{\left\langle\sigma_{\mathrm{ann}}(X X) v_{X}\right\rangle \mathrm{TeV}^{2}}
$$


The TeV scale emerges naturally as the geometric mean of $T_{o} \simeq 2.73 K$ and $m_{P} \sim 1.2 \times 10^{19} \mathrm{GeV}$ ! Using the dimensional estimate $\left\langle\sigma_{\text {ann }}(X X) v_{X}\right\rangle \simeq C \cdot \alpha^{2} / m_{X}^{2}$, where $\alpha$ is a generic coupling strength and $C$ is a model-dependent numerical coefficient, we find

$$
m_{X} \simeq(16 \alpha \sqrt{C}) \sqrt{\frac{\Omega_{X} h^{2}}{0.25}} \mathrm{TeV},
$$

which yields the expectation that $m_{X} \lesssim 1 \mathrm{TeV}$. We will discuss in Section 3 the extent to which this argument implies that the LHC is "guaranteed" to discover a cold dark matter particle. For the moment, we just point out that the above discussion assumed that the particle was at one time in thermal equilibrium, which is not necessarily the case. Counter-examples include the axion discussed here by Turner [3], and the superheavy relic particles discussed here by Kolb [4], and in Section 4 of this talk.

\section{Neutrinos}

Particle physics experiments [5] tell us that neutrino masses must be much smaller than those of the corresponding charged leptons and quarks:

$$
\begin{aligned}
& m_{\nu_{e}} \lesssim 3.5 \mathrm{eV} \text { vs. } m_{e} \simeq 0.511 \mathrm{MeV} \\
& m_{\nu_{\mu}} \lesssim 160 \mathrm{keV} \text { vs. } m_{\mu} \simeq 105 \mathrm{MeV} \\
& m_{\nu_{\tau}} \lesssim 18 \mathrm{MeV} \text { vs. } m_{\tau} \simeq 1.78 \mathrm{GeV}
\end{aligned}
$$

There is another difference between neutrinos and other particles, namely that only left-handed neutrinos are known to exist, produced by the familiar $V-A$ charged current:

$$
J_{\mu}=\bar{e} \gamma_{\mu}\left(1-\gamma_{5}\right) \nu_{e}+\bar{\mu} \gamma_{\mu}\left(1-\gamma_{5}\right) \nu_{\mu}+\bar{\tau} \gamma_{\mu}\left(1-\gamma_{5}\right) \nu_{\tau}
$$

whereas both quarks and charged leptons have both left- and right-handed states $q_{L, R}, \ell_{L, R}$. Thus "Dirac" masses $m^{D}$ coupling them are possible:

$$
g_{h \bar{f} f} H_{\Delta I=1 / 2, \Delta L=0} \bar{f}_{R} f_{L} \Rightarrow m_{f}^{D}=g_{H \bar{f} f}\left\langle 0\left|H_{\Delta I=1 / 2, \Delta L=0}\right| 0\right\rangle,
$$

where the quantum numbers of the Standard Model Higgs have been indicated explicitly. The following puzzles then arise. If right-handed neutrinos $\nu_{R}$ exist, why are the $m_{\nu}$ (8) so small? If $\nu_{R}$ do not exist, can neutrinos acquire masses: $m_{\nu} \neq 0$ ?

Most particle theorists believe that particles should be massless only if there is an exact gauge symmetry to guarantee this, as is the case for the photon and gluon. However, there is no such candidate symmetry to guarantee $m_{\nu}=0$, so most of us expect $m_{\nu} \neq 0$. The fact that the $\nu$ have no exact gauge quantum numbers enables them to have Majorana masses $m^{M}$, since $\bar{f}_{R}$ in (10) is replaced by $\overline{f_{L}^{c}}=f_{L}^{T} C$, where $C$ is an antisymmetric matrix:

$$
m_{\nu}^{M} \bar{\nu}_{L}^{c} \nu_{L}=m_{\nu}^{M} \nu_{L}^{T} C \nu_{L} \equiv m_{\nu}^{M} \nu_{L} \cdot \nu_{L}
$$


This is not possible for quarks and charged leptons, because both $q_{L} \cdot q_{L}$ and $\ell_{l} \cdot \ell_{l}$ have $Q_{e m} \neq 0$, whilst $q_{L} \cdot q_{L}$ also has non-zero colour. Such a Majorana neutrino mass (11) would require lepton-number violation: $\Delta L=2$ and weak isospin $\Delta I=1$, which does contradict any sacred theoretical principles, but is not provided by the Higgs fields in the Standard Model or in the minimal SU(5) GUT. A Majorana mass term (11) could in principle be provided by a suitable "exotic" Higgs field:

$$
g_{H \nu \nu} H_{\Delta I=1, \Delta L=2} \nu_{L} \cdot \nu_{L} \Rightarrow m_{\nu}^{M}=g_{H \nu \nu}\left\langle 0\left|H_{\Delta I=1, \Delta L=2}\right| 0\right\rangle,
$$

as appears in some non-minimal GUT models such as $\mathrm{SO}(10)$ with a 126 Higgs representation. However, there are difficulties with sich a scenario, since one would have expected a $\Delta I=1$ Majoron particle which should have been detected via invisible $Z^{0}$ decays. Alternatively, one can obtain $m_{\nu}^{M}$ from a non-renormalizable coupling to the Standard Model Higgs:

$$
\frac{g_{5}}{M}\left(H_{\Delta I=1 / 2} \nu_{L}\right) \cdot\left(H_{\Delta I=1 / 2} \nu_{L}\right) \Rightarrow m_{\nu}^{M}=g_{5} \frac{\left\langle 0\left|H_{\Delta I=1 / 2}\right| 0\right\rangle^{2}}{M} .
$$

The question then arises: what could be the origin of the large mass parameter $M$ ? The most natural possibility is the exchange of a massive singlet neutrino field, traditionally called a right-handed neutrino $\nu_{R}$, though this nomenclature is somewhat anachronistic. Given a $\nu_{R}$, a Dirac mass $m_{\nu}^{D}=g_{H \bar{\nu} \nu}\left\langle 0\left|H_{\Delta I=1 / 2}\right| 0\right\rangle$ is also possible, and one arrives at the famous see-saw mass matrix [6]:

$$
\left(\nu_{L}, \bar{\nu}_{R}\right)\left(\begin{array}{cc}
0 & m_{\nu}^{D} \\
m_{\nu}^{D} & M
\end{array}\right)\left(\begin{array}{l}
\nu_{L} \\
\bar{\nu}_{R}
\end{array}\right)
$$

One would expect the $M_{\nu}^{D}$ entries in (14) to be of order $m_{q}$ or $m_{\ell} \lesssim 0\left(M_{W}\right)$, so the matrix diagonalization yields the following approximate eigenstates and eigenvalues:

$$
\begin{aligned}
\nu_{L}+0\left(\frac{M_{W}}{M}\right) \bar{\nu}_{R} & : & m_{L}=m_{\nu}^{D} M^{-1}\left(m_{\nu}^{D}\right)^{\dagger}=\mathcal{O}\left(\frac{m_{W}^{2}}{M}\right) \\
\bar{\nu}_{R}+0\left(\frac{m_{W}}{M}\right) \nu_{L} & : & m_{R}=M .
\end{aligned}
$$

Thus there are naturally very light neutrinos if $M \gg m_{W}$, as would be expected in a GUT with $M=\mathcal{O}\left(M_{G U T}\right)$.

If the Dirac masses of the different neutrino generations scale like the corresponding quark or charged-lepton masses, one would expect

$$
m_{\nu_{i}} \simeq \frac{m_{q_{i}}^{2} \text { or } m_{\ell_{i}}^{2}}{M_{i}}
$$

and hence

$$
m_{\nu_{e}} \ll m_{\nu_{\mu}} \ll m_{\nu_{\tau}},
$$

if the heavy Majorana mass matrix is diagonal, and if its eigenvalues $M_{i}$ are approximately the same. As an example, putting $m_{\nu}^{D} \sim 100 \mathrm{GeV}$ for the third generation, one finds $m_{\nu_{3}} \sim 0.1 \mathrm{eV}$ if $M_{3} \sim 10^{14} \mathrm{GeV}$. Before the advent of the atmospheric neutrino data [7, 8, 9], one might 
also have expected small neutrino mixing angles, analogous to those for quarks, which originate from Dirac mass matrices.

This and the prejudice (17) are not necessarily supported by the atmospheric neutrino data $[7,8,9]$, which suggest a large mixing angle: $\sin ^{2} 2 \theta_{\mu \tau} \gtrsim 0.8$ and a mass-squared difference $\Delta m_{\text {atmo }}^{2} \sim\left(10^{-2}\right.$ to $\left.10^{-3}\right) \mathrm{eV}^{2}$. We also recall that the solar neutrino data $[10,11]$ favour one of three possible solutions: the large-angle MSW solution with

$$
\Delta m_{\text {solar }}^{2} \sim 10^{-5} \mathrm{eV}^{2}, \quad \sin ^{2} 2 \theta \gtrsim 0.8
$$

the small-angle MSW solution with

$$
\Delta m_{\text {solar }}^{2} \sim 10^{-5} \mathrm{eV}^{2}, \quad \sin ^{2} 2 \theta \sim 10^{-2},
$$

or vacuum oscillations with

$$
\Delta m_{\text {solar }}^{2} \sim 10^{-10} \mathrm{eV}^{2}, \quad \sin ^{2} 2 \theta \gtrsim 0.6
$$

Which of these solutions might be favoured by post-super-Kamiokande models of neutrino masses, and how plausible is neutrino hot dark matter of cosmological and astrophysical significance, which would require $m_{\nu} \gtrsim 1 \mathrm{eV}$ for at least one neutrino species?

A first comment is that large neutrino mixing is (in retrospect) not at all implausible [12]. For one thing, it is not at all necessary that $m_{\nu}^{D} \propto m_{q}$ or $m_{\ell}$. For example, in a specific flipped $\mathrm{SU}(5)$ model used earlier to discuss quark and lepton masses, we found [13]

$$
m_{\nu}^{D} \sim\left(\begin{array}{ccc}
\mathcal{O}(\eta) & \mathcal{O}(1) & 0 \\
\mathcal{O}(\eta) & \mathcal{O}(1) & 0 \\
0 & 0 & \mathcal{O}(1)
\end{array}\right)
$$

where $\eta$ is a small parameter, which would yield at least one large neutrino mixing angle. Moreover, there is no good reason why the heavy singlet Majorana mass matrix should be (approximately) diagonal in the same basis. For example, in models with a $U(1)$ flavour symmetry one expects matrix elements

$$
M_{i j} \propto \epsilon^{n_{i}+n_{j}} M_{G U T}
$$

where $\epsilon$ is an expansion parameter. If $n_{i}=-n_{j}$, one gets a large off-diagonal entry $M_{i j}=$ $\mathcal{O}(1) \times M_{G U T}$, which looks at least as plausible as having $n_{i}=0$, which would be required to give a large diagonal entry $M_{i i}=\mathcal{O}\left(M_{G U T}\right)$. Such a large off-diagonal entry would be another source of large neutrino mixing.

With so many different features contributing to the light neutrino mass matrix, there is no obvious symmetry or other reason why near-degeneracy $m_{i} \sim m_{j} \gg\left|m_{i}-m_{j}\right|$ should occur. Therefore, we expect that there may be a hierarchy of masses:

$$
m_{3} \sim \sqrt{\Delta m_{\text {atmo }}^{2}}>m_{2} \sim \sqrt{\Delta m_{\text {solar }}^{2}}>m_{1}
$$

If this is indeed the case, even the heaviest light neutrino would weigh $\lesssim 0.1 \mathrm{eV}$, and would not be of great astrophysical and cosmological interest [14]. 


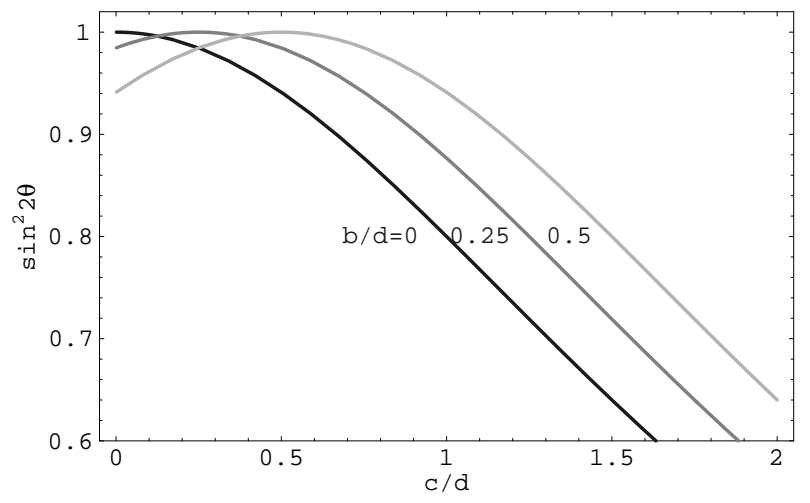

Figure 1: Light-neutrino mixing in a simple $2 \times 2$ model (22), as a function of the ratios of mass-matrix elements [13].

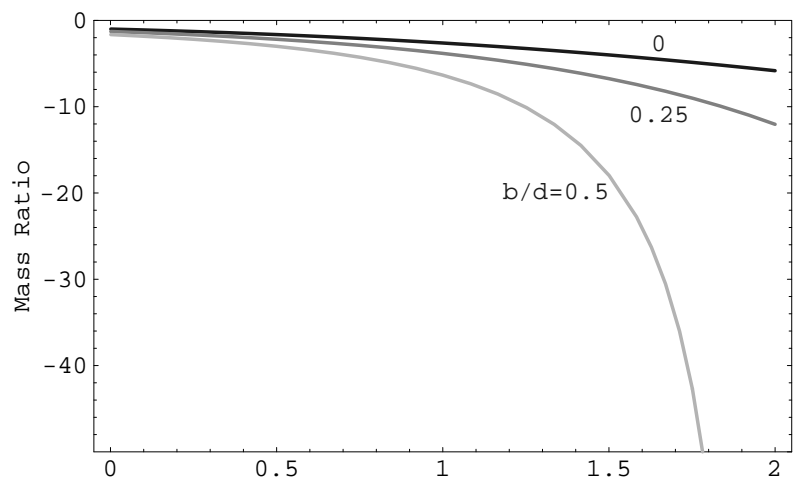

Figure 2: Light-neutrino mass hierarchy in a simple $2 \times 2$ model (22), as a function of the ratios of mass-matrix elements [13].

A corollary question is the extent to which large mixing is compatible with a large neutrino mass hierarchy. To address this, we may consider a simple two-state model [13]

$$
m_{\nu}=\left(\begin{array}{ll}
b & d \\
d & c
\end{array}\right)
$$

and diagonalize it to obtain eigenvalues $m_{2}$ and $m_{3}$ and a mixing angle $\theta_{23}$. We see in Figs. 1 and 2 that a hierarchy $m_{3}=\mathcal{O}(10) m_{2}$ is quite compatible with large mixing $\sin ^{2} \theta_{23}$ without fine-tuning of the ratios $b / d, c / d$, for example if $b / d \sim 0.5$ and $c / d \sim 1.5$. Moreover, the lightneutrino mixing angle may be enhanced by renormalization-group effects between the GUT and electroweak scales [15]:

$$
16 \pi^{2} \frac{d}{d t}\left(\sin ^{2} \theta_{23}\right)=-2\left(\sin ^{2} \theta_{23}\right)\left(\cos ^{2} \theta_{23}\right) \times\left(\lambda_{3}^{2}-\lambda_{2}^{2}\right) \frac{m_{33}+m_{22}}{m_{33}-m_{22}}
$$

as seen in Fig. 3. The renormalization-group enhancement is particularly important if $\lambda_{3}^{2}-\lambda_{2}^{2}$ is large, as may happen at large $\tan \beta$ in supersymmetric models, and/or if the diagonal etries $m_{33}$ 


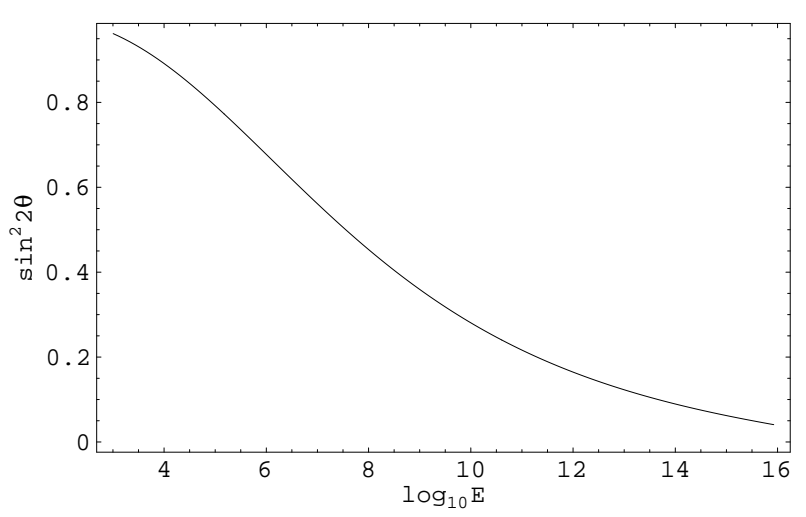

Figure 3: Possible renormalization-group running of the light-neutrino mixing angle [13].

and $m_{22}$ are almost equal. We infer that the hierarchy (21) is plausible if $\Delta m_{\text {solar }}^{2} \sim 10^{-5} \mathrm{eV}^{2}$ as in the large- and small-angle MSW solutions (18a,b). However, it seems difficult to stretch the hierarchy (21) as far as would be required by the vacuum solution (18c) to the solar neutrino problem.

Our present inclination is therefore to favour

$$
m_{\nu_{3}} \sim\left(10^{-1} \text { to } 10^{-1 \frac{1}{2}}\right) \mathrm{eV}, m_{2} \sim 10^{-2} \frac{1}{2} \mathrm{eV}
$$

and the remaining question is whether the large- or small-angle MSW solution $(18 \mathrm{a}, \mathrm{b})$ is to be favoured. In our specific flipped $S U(5)$ model [13], we found not only large off-diagonal entries in $m_{\nu}^{D}$ (19), but also large off-diagonal entries (20) in the singlet-neutrino mass matrix:

$$
M \sim\left(\begin{array}{ccc}
X & X & 0 \\
X & 0 & X \\
0 & X & X
\end{array}\right)
$$

where all of the indicated non-zero entries might a priori be comparable in magnitude. We therefore find the large-angle MSW solution (18a) to be at least as plausible as the small-angle MSW solution (18b), perhaps even more so. A final comment concerns the magnitudes of the entries in (25): we estimate their natural order of magnitude to be $0\left(10^{13 \pm 2}\right) \mathrm{GeV}$, corresponding to $m_{\nu_{3}}=10^{0 \pm 2} \mathrm{eV}$, overlapping comfortably with the desired range (21).

In my view, although the super-Kamiokande data [7] make a very strong case for atmospheric neutrino oscillations, particle physicists will not be completely convinced until they have verified the effect using a beam with controllable energy, spectrum and flavour content, as provided by an accelerator neutrino beam. One such project, the K2K experiment [16], is to start taking data in 1999, and another, the NUMI/MINOS project [17], has also been approved. A third project, neutrino Gran Sasso (NGS), has been studied by a joint CERN-INFN working group [18], and is ready for construction if funding is approved. The K2K project is at relatively low energy, insufficient to produce the $\tau$ lepton. The NUMI/MINOS and NGS projects are at higher energies, and should be able to reach down to $\Delta m^{2} \sim 10^{-3} \mathrm{eV}^{2}$, though without much margin. Nevertheless, in a few years we may expect to know whether accelerator experiments confirm 
the super-Kamiokande results. However, as mentioned earlier, it currently seems unlikely that neutrinos will turn out to be an important component of the dark matter [14].

\section{The Lightest Supersymmetric Particle}

The motivation for supersymmetry at an accessible energy is provided by the gauge hierarchy problem [19], namely that of understanding why $m_{W} \ll m_{P}$, the only candidate for a fundamental mass scale in physics. Alternatively and equivalently, one may ask why $G_{F} \sim g^{2} / m_{W}^{2} \gg$ $G_{N}=1 / m_{P}^{2}$, where $M_{P}$ is the Planck mass, expected to be the fundamental gravitational mass scale. Or one may ask why the Coulomb potential inside an atom is so much larger than the Newton potential, which is equivalent to why $e^{2}=\mathcal{O}(1) \gg m_{p} m_{e} / m_{P}^{2}$, where $m_{p, e}$ are the proton and electron masses.

One might think it would be sufficient to choose the bare mass parameters: $m_{W} \ll m_{P}$. However, one must then contend with quantum corrections, which are quadratically divergent:

$$
\delta m_{H, W}^{2}=\mathcal{O}\left(\frac{\alpha}{\pi}\right) \Lambda^{2}
$$

which is much larger than $m_{W}$, if the cutoff $\Lambda$ representing the appearance of new physics is taken to be $\mathcal{O}\left(m_{P}\right)$. This means that one must fine-tune the bare mass parameter so that it is almost exactly cancelled by the quantum correction (26) in order to obtain a small physical value of $m_{W}$. This seems unnatural, and the alternative is to introduce new physics at the $\mathrm{TeV}$ scale, so that the correction (26) is naturally small.

At one stage, it was proposed that this new physics might correspond to the Higgs boson being composite [20]. However, calculable scenarios of this type are inconsistent with the precision electroweak data from LEP and elsewhere. The alternative is to postulate approximate supersymmetry [21], whose pairs of bosons and fermions produce naturally cancelling quantum corrections:

$$
\delta m_{W}^{2}=\mathcal{O}\left(\frac{\alpha}{\pi}\right)\left|m_{B}^{2}-m_{F}^{2}\right|
$$

that are naturally small: $\delta m_{W}^{2} \lesssim m_{W}^{2}$ if

$$
\left|m_{B}^{2}-m_{F}^{2}\right| \lesssim 1 \mathrm{TeV}^{2}
$$

There are many other possible motivations for supersymmetry, but this is the only one that gives reason to expect that it might be accessible to the current generation of accelerators and in the range (7) expected for a cold dark matter particle.

The minimal supersymmetric extension of the Standard Model (MSSM) has the same gauge interactions as the Standard Model, and the Yukawa interactions are very similar:

$$
\lambda_{d} Q D^{c} H+\lambda_{\ell} L E^{c} H+\lambda_{u} Q U^{c} \bar{H}+\mu \bar{H} H
$$

where the capital letters denote supermultiplets with the same quantum numbers as the lefthanded fermions of the Standard Model. The couplings $\lambda_{d, \ell, u}$ give masses to down quarks, 
leptons and up quarks respectively, via distinct Higgs fields $H$ and $\bar{H}$, which are required in order to cancel triangle anomalies. The new parameter in (19) is the bilinear coupling $\mu$ between these Higgs fields, that plays a significant rôle in the description of the lightest supersymmetric particle, as we see below. The gauge quantum numbers do not forbid the appearance of additional couplings

$$
\lambda L L E^{c}+\lambda^{\prime} L Q D^{c}+\lambda U^{c} D^{c} D^{c}
$$

but these violate lepton or baryon number, and we assume they are absent. One significant aspect of the MSSM is that the quartic scalar interactions are determined, leading to important constraints on the Higgs mass, as we also see below.

Supersymmetry must be broken, since supersymmetric partner particles do not have identical masses, and this is usually parametrized by scalar mass parameters $m_{0_{i}}^{2}\left|\phi_{i}\right|^{2}$, gaugino masses $\frac{1}{2} M_{a} \tilde{V}_{a} \cdot \tilde{V}_{a}$ and trilinear scalar couplings $A_{i j k} \lambda_{i j k} \phi_{i} \phi_{j} \phi_{k}$. These are commonly supposed to be inputs from some high-energy physics such as supergravity or string theory. It is often hypothesized that these inputs are universal: $m_{0_{i}} \equiv m_{0}, M_{a} \equiv M_{1 / 2}, A_{i j k} \equiv A$, but these assumptions are not strongly motivated by any fundamental theory. The physical sparticle mass parameters are then renormalized in a calculable way:

$$
m_{0_{i}}^{2}=m_{0}^{2}+C_{i} m_{1 / 2}^{2}, \quad M_{a}=\left(\frac{\alpha_{a}}{\alpha_{G U T}}\right) m_{1 / 2}
$$

where the $C_{i}$ are calculable coefficients [22] and MSSM phenomenology is then parametrized by $\mu, m_{0}, m_{1 / 2}, A$ and $\tan \beta$ (the ratio of Higgs v.e.v.'s).

Precision electroweak data from LEP and elsewhere provide two qualitative indications in favour of supersymmetry. One is that the inferred magnitude of quantum corrections favour a relatively light Higgs boson [23]

$$
m_{h}=66_{-39}^{+74} \pm 10 \mathrm{GeV}
$$

which is highly consistent with the value predicted in the MSSM: $m_{h} \lesssim 150 \mathrm{GeV}[24]$ as a result of the constrained quartic couplings. (On the other hand, composite Higgs models predicted an effective Higgs mass $\gtrsim 1 \mathrm{TeV}$ and other unseen quantum corrections.) The other indication in favour of low-energy supersymmetry is provided by measurements of the gauge couplings at LEP, that correspond to $\sin ^{2} \theta_{W} \simeq 0.231$ in agreement with the predictions of supersymmetric GUTs with sparticles weighing about $1 \mathrm{TeV}$, but in disagreement with nonsupersymmetric GUTs that predict $\sin ^{2} \theta_{W} \sim 0.21$ to 0.22 [25]. Neither of these arguments provides an accurate estimate of the sparticle mass scales, however, since they are both only logarithmically sensitive to $m_{0}$ and/or $m_{1 / 2}$.

The lightest supersymmetric particle (LSP) is expected to be stable in the MSSM, and hence should be present in the Universe today as a cosmological relic from the Big Bang [26]. This is a consequence of a multiplicatively-conserved quantum number called $R$ parity, which is related to baryon number, lepton number and spin:

$$
R=(-1)^{3 B+L+2 S}
$$


It is easy to check that $R=+1$ for all Standard Model particles and $R=-1$ for all their supersymmetric partners. The interactions (30) would violate $R$, but not a Majorana neutrino mass term or the other interactions in $S U(5)$ or $S O(10)$ GUTs. There are three important consequences of $R$ conservation: (i) sparticles are always produced in pairs, e.g., $p p \rightarrow \tilde{q} \tilde{g} X$, $e^{+} e^{-} \rightarrow \tilde{\mu}^{+} \tilde{\mu}^{-}$, (ii) heavier sparticles decay into lighter sparticles, e.g., $\tilde{q} \rightarrow q \tilde{g}, \tilde{\mu} \rightarrow \mu \tilde{\gamma}$, and (iii) the LSP is stable because it has no legal decay mode.

If such a supersymmetric relic particle had either electric charge or strong interactions, it would have condensed along with ordinary baryonic matter during the formation of astrophysical structures, and should be present in the Universe today in anomalous heavy isotopes. These have not been seen in studies of $H, H e, B e, L i, O, C, N a, B$ and $F$ isotopes at levels ranging from $10^{-11}$ to $10^{-29}$ [27], which are far below the calculated relic abundances from the Big Bang:

$$
\frac{n_{\text {relic }}}{n_{p}} \gtrsim 10^{-6} \text { to } 10^{-10}
$$

for relics with electromagnetic or strong interactions. Except possibly for very heavy relics, one would expect these primordial relic particles to condense into galaxies, stars and planets, along with ordinary bayonic material, and hence show up as an anaomalous heavy isotope of one or more of the elements studied. There would also be a 'cosmic rain' of such relics [28], but this would presumably not be the dominant source of such particles on earth. The conflict with (34) is sufficiently acute that the lightest supersymmetric relic must presumably be electromagnetically neutral and weakly interacting [26]. In particular, I believe that the possibility of a stable gluino can be excluded. This leaves as scandidates for cold dark matter a sneutrino $\tilde{\nu}$ with spin 0 , some neutralino mixture of $\tilde{\gamma} / \tilde{H}^{0} / \tilde{Z}$ with spin $1 / 2$, and the gravitino $\tilde{G}$ with spin $3 / 2$.

LEP searches for invisible $Z^{0}$ decays require $m_{\tilde{\nu}} \gtrsim 43 \mathrm{GeV}$ [29], and searches for the interactions of relic particles with nuclei then enforce $m_{\tilde{\nu}} \gtrsim$ few $\mathrm{TeV}$ [30], so we exclude this possibility for the LSP. The possibility of a gravitino $\tilde{G}$ LSP has attracted renewed interest recently with the revival of gauge-mediated models of supersymmetry breaking [31], and could constitute warm dark matter if $m_{\tilde{G}} \simeq 1 \mathrm{keV}$. In this talk, however, I concentrate on the $\tilde{\gamma} / \tilde{H}^{0} / \tilde{Z}^{0}$ neutralino combination $\chi$, which is the best supersymmetric candidate for cold dark matter.

The neutralinos and charginos may be characterized at the tree level by three parameters: $m_{1 / 2}, \mu$ and $\tan \beta$. The lightest neutralino $\chi$ simplifies in the limit $m_{1 / 2} \rightarrow 0$ where it becomes essentially a pure photino $\tilde{\gamma}$, or $\mu \rightarrow 0$ where it becomes essentially a pure higgsino $\tilde{H}$. These possibilities are excluded, however, by LEP and the FNAL Tevatron collider [29]. From the point of view of astrophysics and cosmology, it is encouraging that there are generic domains of the remaining parameter space where $\Omega_{\chi} h^{2} \simeq 0.1$ to 1 , in particular in regions where $\chi$ is approximately a $U(1)$ gaugino $\tilde{B}$, as seen in Fig. 4 [32].

Purely experimental searches at LEP enforce $m_{\chi} \gtrsim 30 \mathrm{GeV}$, as seen in Fig. 5 [33]. This bound can be strengthened by making various theoretical assumptions, such as the universality of scalar masses $m_{0_{i}}$, including in the Higgs sector, the cosmological dark matter requirement that $\Omega_{\chi} h^{2} \leq 0.3$ and the astrophysical preference that $\Omega_{\chi} h^{2} \geq 0.1$. Taken together as in Fig. 


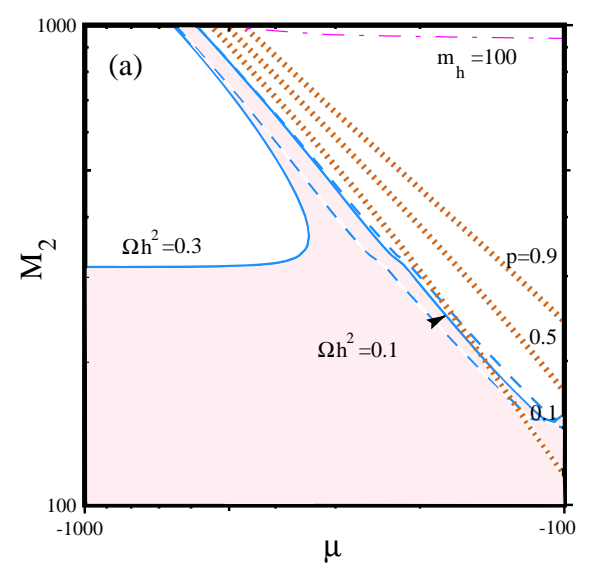

(a)

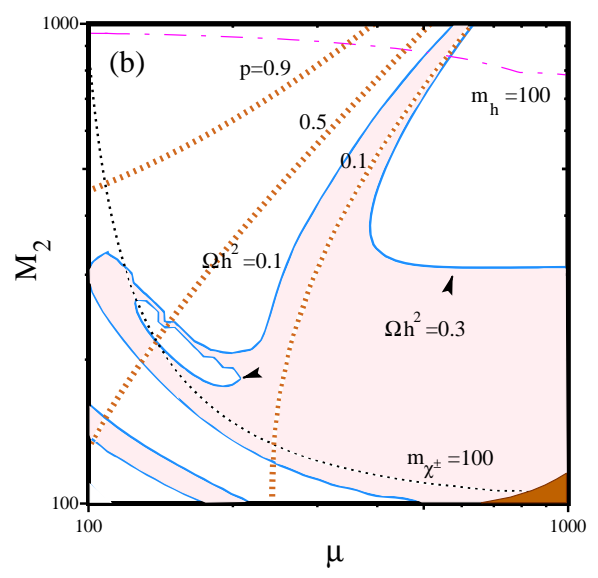

(b)

Figure 4: Regions of the $\left(\mu, M_{2}\right)$ plane in which the supersymmetric relic density may lie within the interesting range $0.1 \leq \Omega h^{2} \leq 0.3$ [32]. 


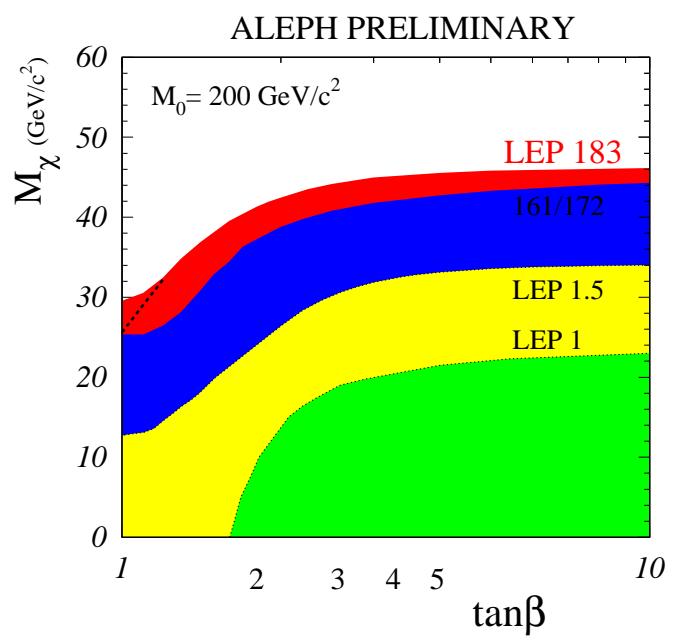

Figure 5: Experimental lower limit on the lightest neutralino mass, inferred from unsuccessful chargino and neutralino searches at LEP [33].
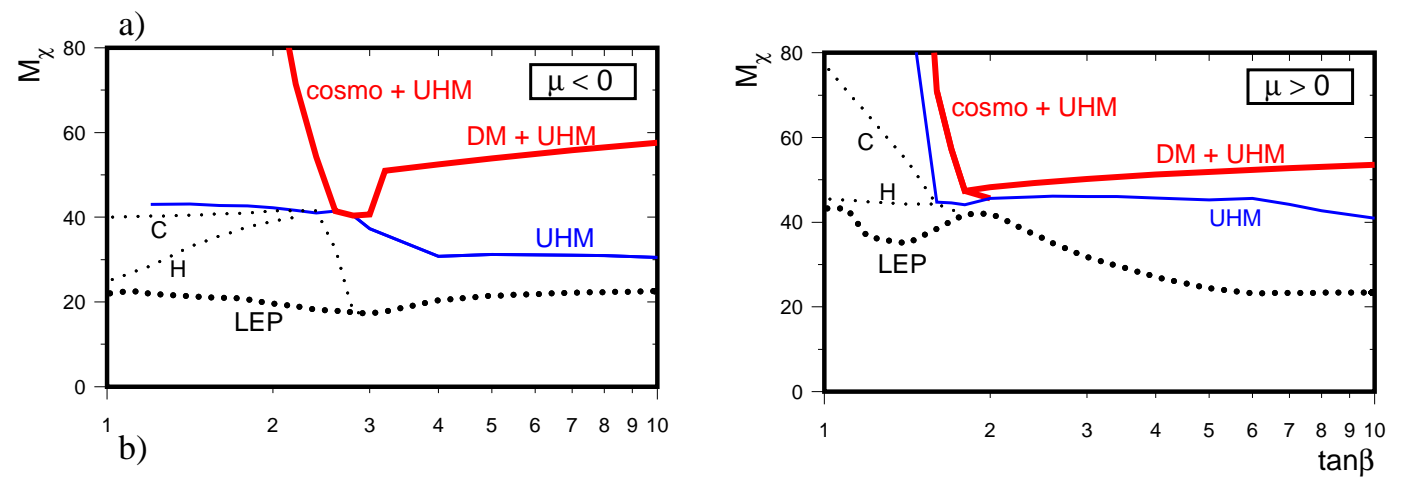

Figure 6: Theoretical lower limits on the lightest neutralino mass, obtained by using the unsuccessful Higgs searches ( $H)$, the cosmological upper limit on the relic density $(C)$, the assumption that all input scalar masses are universal, including those of the Higgs multiplets (UHM), and combining this with the cosmological upper (cosmo) and astrophysical lower (DM) limits on the cold dark matter density [29]. 

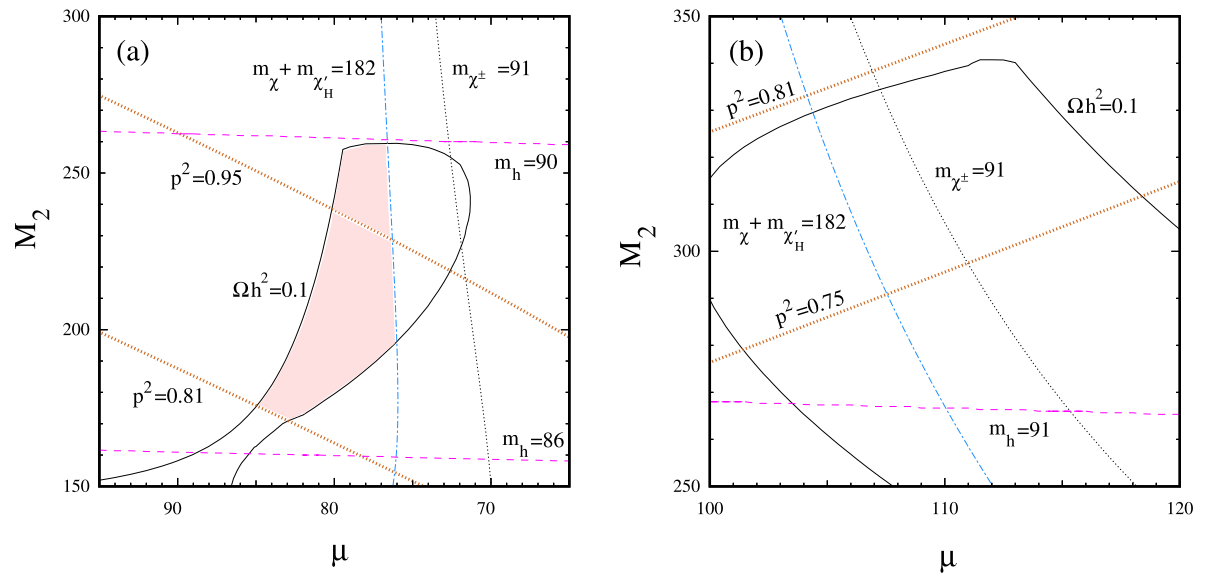

Figure 7: The regions of the $\left(\mu, M_{2}\right)$ plane where the lightest supersymmetric particle may still be a Higgsino, taking into account the indicated LEP constraints [32]. The Higgsino purity is indicated by $p^{2}$.

6 , we see that they enforce

$$
m_{\chi} \gtrsim 42 \mathrm{GeV}
$$

and LEP should eventually be able to establish or exclude $m_{\chi}$ up to about $50 \mathrm{GeV}$. As seen in Fig. 7, LEP has already explored almost all the parameter space available for a Higgsino-like LSP, and this possibility will also be thoroughly explored by LEP [33].

Should one be concerned that no sparticles have yet been seen by either LEP or the FNAL Tevatron collider? One way to quantify this is via the amount of fine-tuning of the input parameters required to obtain the physical value of $m_{W}[34]$ :

$$
\Delta_{o}=\operatorname{Max}_{i}\left|\frac{a_{i}}{m_{W}} \frac{\partial m_{W}}{\partial a_{i}}\right|
$$

where $a_{i}$ is a generic supergravity input parameter. As seen in Fig. 8, the LEP exclusions impose [35]

$$
\Delta_{o} \gtrsim 8
$$

Although fine-tuning is a matter of taste, this is perhaps not large enough to be alarming, and could in any case be reduced significantly if a suitable theoretical relation between some input parameters is postulated [35]. It is interesting to note that the amount of fine-tuning $\Delta_{o}$ is minimized when $\Omega_{\chi} h^{2} \sim 0.1$ as preferred astrophysically, as seen in Fig. 9 [36]. This means that solving the gauge hierarchy problem naturally leads to a relic neutralino density in the range of interest to astrophysics and cosmology. I am unaware of any analogous argument for the neutrino or the axion.

As $m_{\chi}$ increases, the LSP annihilation cross-section decreases and hence its relic number and mass density increase. How heavy could the LSP be? Until recently, the limit given was $m_{\chi} \lesssim 300 \mathrm{GeV}$ [37]. However, it has now been pointed out that there are regions of the MSSM parameter space where co-annihilations of the $\chi$ with the stau slepton $\tilde{\tau}$ could be important, as 

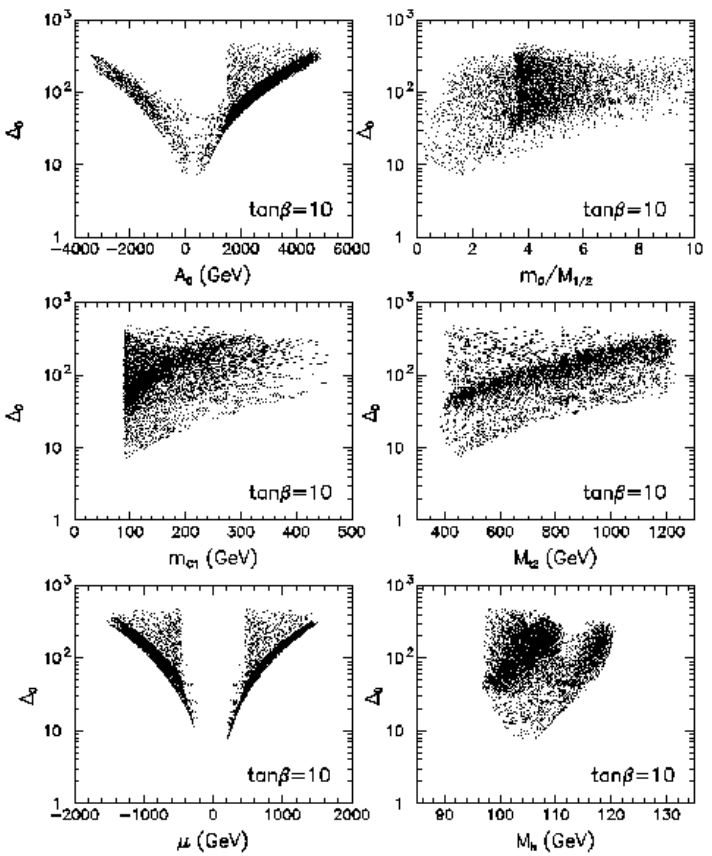

Figure 8: The fine-tuning price $\Delta_{0}$ imposed by LEP for $\tan \beta=10$, as a function of model parameters [35].
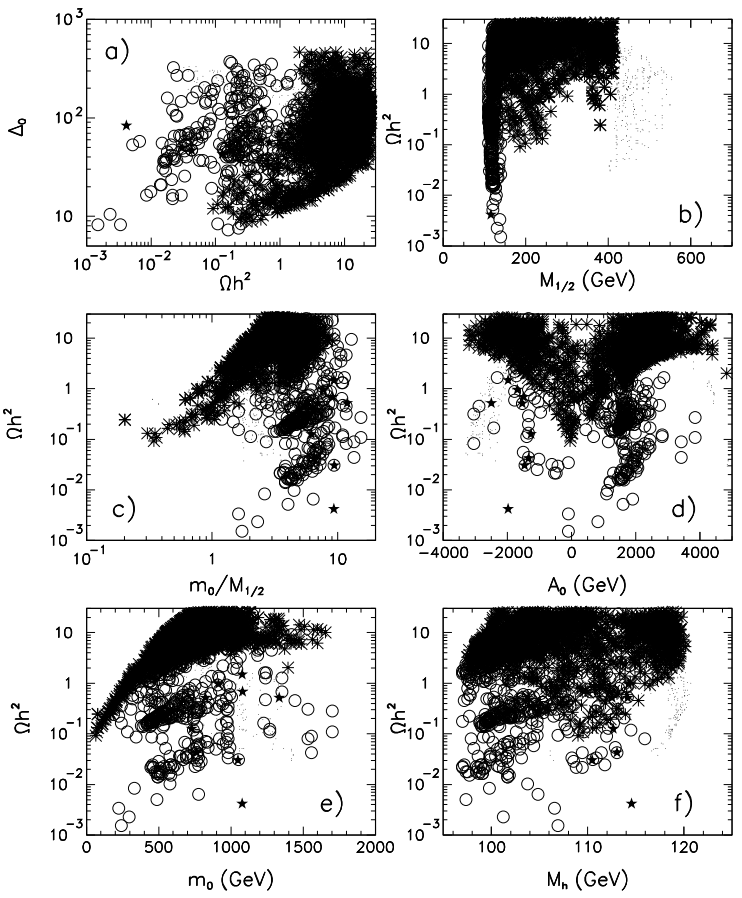

Figure 9: The correlation between the fine-tuning price $\Delta_{0}$ and the relic density $\Omega h^{2}$, showing dependences on model parameters [36]. 

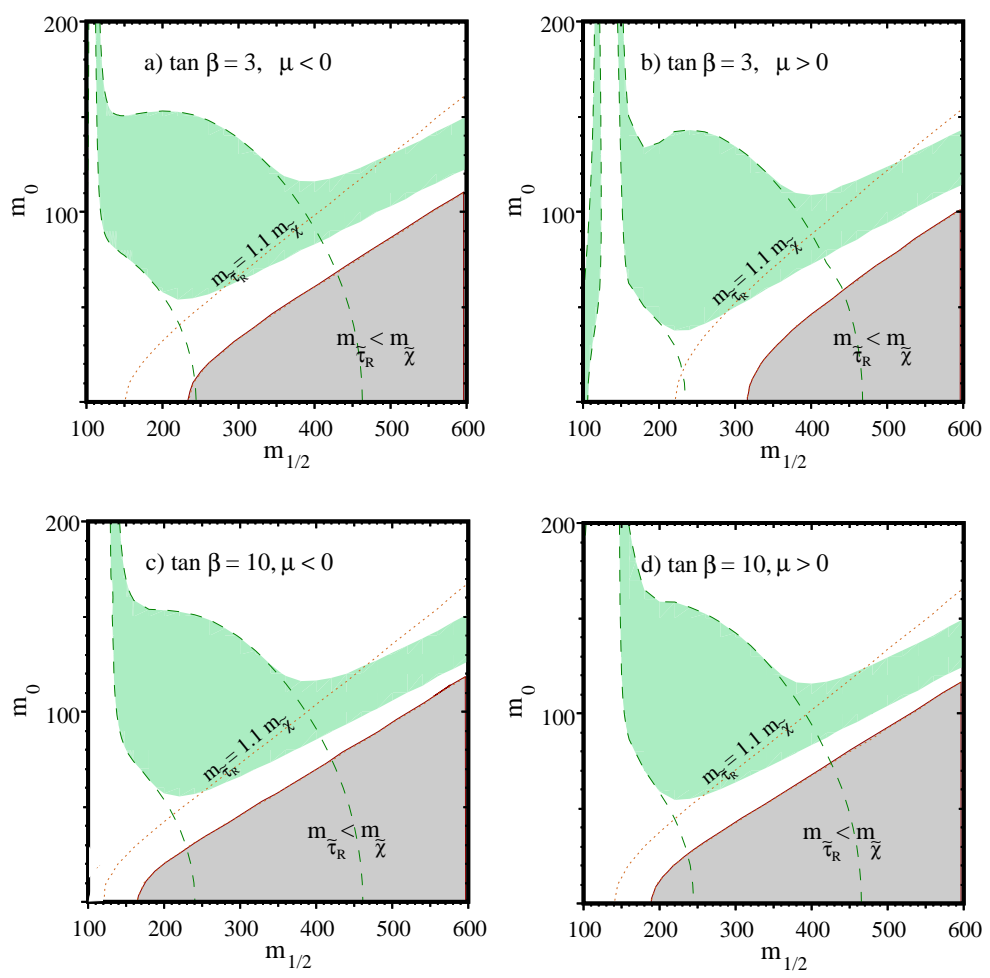

Figure 10: The change in the domain of parameter space allowed by the requirements $0.1 \leq$ $\Omega h^{2} \leq 0.3$ after (shaded region) and before (dashed lines) including $\tilde{\tau}$ co-annihilation [38]. 


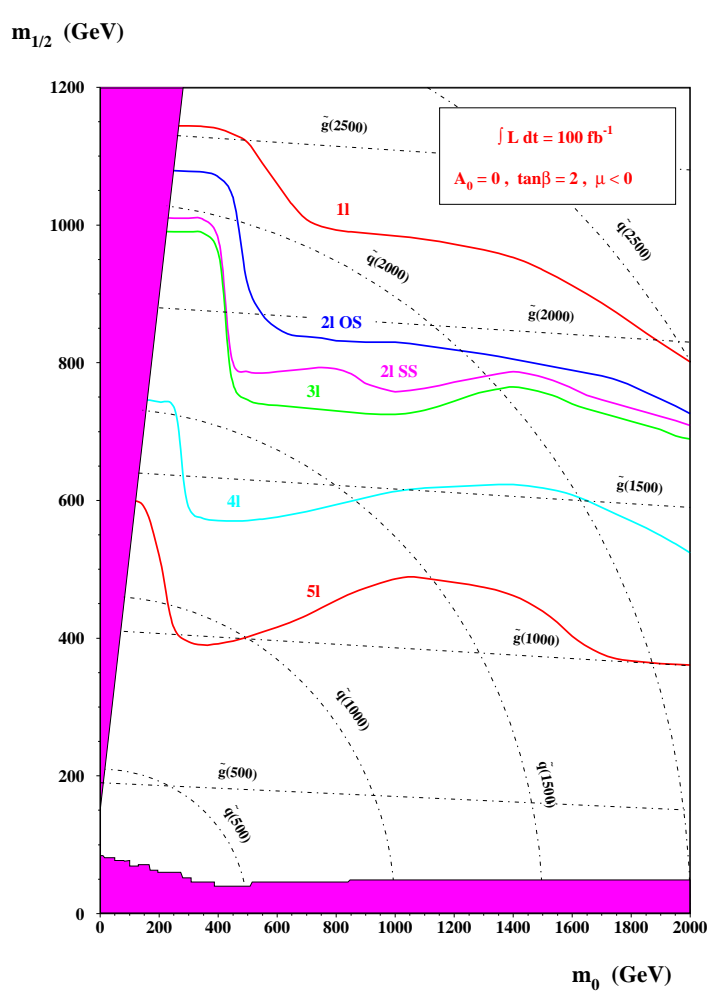

Figure 11: The region of the $\left(m_{0}, m_{1 / 2}\right)$ plane accessible to sparticle searches at the LHC [40].

seen in Fig. 10 [38]. These co-annihilations would suppress $\Omega_{\chi}$, allowing a heavier neutralino mass, and we now find that [38]

$$
m_{\chi} \lesssim 600 \mathrm{GeV}
$$

is possible. In the past, it was thought that all the cosmologically-preferred region of MSSM parameter space ${ }^{1}$ could be explored by the LHC [40], as seen in Fig. 11, but it now seems possible that there may be a delicate region close to the upper bound (38). This point requires further study.

\section{Superheavy Relic Particles}

The expectation (7), exemplified by the MSSM range (38), was based on the assumption that the cold dark matter particles were at one time in thermal equilibrium. As discussed here by Kolb [4], much heavier relic particles are possible if one invokes non-thermal production mechanisms. Non-thermal decays of inflatons in conventional models of cosmological inflation could yield $\Omega_{\chi} \sim 1$ for $m_{\chi} \sim 10^{13} \mathrm{GeV}$. Preheating via the parametric resonance decay of the

\footnotetext{
${ }^{1}$ There has recently been progress in implementing the constraints from the absence of charge and colourbreaking minima [39].
} 
inflaton could even yield $\Omega_{\chi} \sim 1$ for $m_{\chi} \sim 10^{15} \mathrm{GeV}$. Other possibilities include a first-order phase transition at the end of inflation, and gravitational relic production induced by the rapid change in the scale factor in the early Universe [41]. It is therefore of interest to look for possible experimental signatures of superheavy dark matter.

One such possibility is offered by ultra-high-energy cosmic rays. Those coming from distant parts of the Universe $(D \gtrsim 100 M p c)$ are expected to be cut off at an energy $E \lesssim 5 \times 10^{19} \mathrm{GeV}$, because of the reaction $p+\gamma_{C M B R} \rightarrow \Delta^{+}[42]$. However, no such Greisen-Zatsepin-Kuzmin cut-off is seen in the data! [43] The ultra-high-energy cosmic rays must originate nearby, and should point back to any point-like sources such as AGNs. However, no such sources have been seen.

Could the ultra-high-energy cosmic rays be due to the decays of superheavy relic particles? These should be clustered in galactic haloes (including our own), and hence give an anisotropic flux [44], but there would be no obvious point sources. There have been some reports of anisotropies in high-energy cosmic rays, but it is not clear whether they could originate in superheavy relic decays.

We have analyzed [45] recently possible superheavy relic candidates in string [46] and/or $M$ theory. One expects Kaluza-Klein states when six excess dimensions are compactified: $10 \rightarrow 4$ or $11 \rightarrow 5$, which we call hexons. However, these are expected to weigh $\gtrsim 10^{16} \mathrm{GeV}$, which may be too heavy, and there is no particular reason to expect hexons to be metastable. In $M$ theory, one expects massive states associated with a further compactification: $5 \rightarrow 4$ dimensions, which we call pentons. Their mass could be $\sim 10^{13} \mathrm{GeV}$, which would be suitable, but there is again no good reason to expect them to be metastable. We are left with bound states from the hidden sector of string/ $M$ theory, which we call cryptons [46]. These could also have masses $\sim 10^{13}$ $\mathrm{GeV}$, and might be metastable for much the same reason as the proton in a GUT, decaying via higher-dimensional multiparticle operators. For example, in a flipped $S U(5)$ model we have a hidden-sector $S U(4) \times S O(10)$ gauge group, and the former factor confines four-constituent states which we call tetrons. Initial studies [46, 45] indicate that the lightest of these might well have a lifetime $\gtrsim 10^{17} \mathrm{y}$, which would be suitable for the decays of superheavy dark matter particles. Detailed simulations have been made of the spectra of particles produced by the fragmentation of their decay products [47, 48], and the ultra-high-energy cosmic-ray data are consistent with the decays of superheavy relics weighing $\sim 10^{12} \mathrm{GeV}$, as seen in Fig. 12 [48]. Issues to be resolved here include the roles of supersymmetric particles in the fragmentation cascades, and the relative fluxes of $\gamma, \nu$ and $p$ among the ultra-high-energy cosmic rays.

\section{$5 \quad$ Vacuum Energy}

Data on large-scale structure [49] and high-redshift supernovae [50] have recently converged on the suggestion that the energy of the vacuum may be non-zero. In my view, this represents a wonderful opportunity for theoretical physics: a number to be calculated in the Theory of Everything including quantum gravity. The possibility that the vacuum energy may be non- 


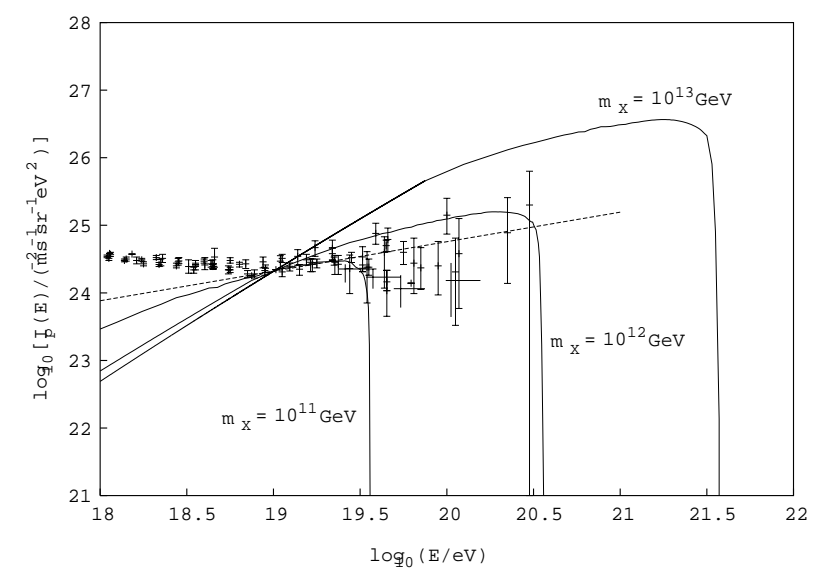

Figure 12: The ultra-high energy cosmic ray flux compared with a model calculation based on the decays of superheavy relic particles [48].

zero may even appear more natural than a zero value, since there is no obvious symmetry or other reason known why it should vanish.

In the above paragraph, I have used the term vacuum energy rather than cosmological constant, because it may not actually be constant. This option has been termed quintessence here by Steinhardt [51], who has discussed a classical scalar-field model that is not strongly motivated by the Standard Model, supersymmetry or GUTs, though something similar might emerge from string theory. I prefer to think that a varying vacuum energy might emerge from a quantum theory of gravity, as the vacuum relaxes towards an asymptotical value (zero?) in an infinitely large and old Universe. We have recently given [52] an example of one such possible effect which yields a contribution to the vacuum energy that decreases as $1 / t^{2}$. This is compatible with the high-redshift supernova data, and one may hope that these could eventually discriminate between such a possibility and a true cosmological constant.

\section{References}

[1] K. Griest, G. Jungman and M. Kamionkowski, Phys.Rept. 267 (1996) 195.

[2] S. Dimopoulos, Phys.Lett. B246 (1990) 347.

[3] M.S. Turner, talk at this meeting; see also astro-ph/9811364, astro-ph/9811366, astro-ph/9811447, astro-ph/9811454.

[4] E.W. Kolb, talk at this meeting; see also hep-ph/9810362.

[5] Particle Data Group, Particle Data Group, C. Caso et al., Eur.Phys.J. C3 (1998) 1.

[6] T. Yanagida, Proc. Workshop on the Unified Theory and the Baryon Number in the Universe (KEK, Japan, 1979);

R. Slansky, Talk at the Sanibel Symposium, Caltech preprint CALT-68-709 (1979). 
[7] Super-Kamiokande collaboration, Y. Fukuda et al., Phys.Rev.Lett. 81 (1998) 1562.

[8] T. Kafka for the Soudan II collaboration, Nucl.Phys.Proc.Suppl. 70 (1999) 340.

[9] MACRO collaboration, M. Ambrosio et al., Phys.Lett. B434 (1998) 451.

[10] J.N. Bahcall, talk at this meeting; see also astro-ph/9808162;

J. N. Bahcall, P. I. Krastev and A. Yu. Smirnov, Phys.Rev. D58 (1998) 096016.

[11] E. Lisi, talk at this meeting; see also

G.L. Fogli, E. Lisi, A. Marrone and G. Scioscia, hep-ph/9808205.

[12] For some recent work, see:

S.F. King, hep-ph/9806440;

J.K. Elwood, N. Igres and P. Ramond, hep-ph/9807325;

V. Barger et al, hep-ph/9806328;

P. Osland and G. Vigdel, hep-ph/9806339;

A. Joshipura and A. Smirnov, hep-ph/9806376;

M. Tanimoto, hep-ph/9807283;

G. Lazarides and N. Vlachos, hep-ph/9807253;

V. Barger, T. Weiler and K. Whisnant, hep-ph/9807319;

J. Pati, hep-ph/9807315;

M. Gonzalez-Carcia et al, hep-ph/9807305;

G. Altarelli and F. Feruglio, hep-ph/9807353.

[13] J. Ellis, G. Leontaris, S. Lola and D.V. Nanopoulos, hep-ph/9808251.

[14] However, combinations of structure-formation and cosmic microwave-background measurements may be sensitive to smaller neutrino masses: M. Tegmark, talk at this meeting; see also

W. Hu, D.J. Eisenstein, M. Tegmark and M. White, astro-ph/9806362.

[15] M. Tanimoto, Phys.Lett. B360 (1995) 41.

[16] Y. Oyama, for the K2K collaboration, hep-ex/9803014.

[17] MINOS collaboration, E. Ables et al., Fermilab Proposal P-875 (1995).

[18] G. Acquistapace et al., CERN report 98-02.

[19] L. Maiani, Proc. Summer School on Particle Physics, Gif-sur-Yvette, 1979 (IN2P3, Paris, 1980) p. 3;

G 't Hooft, in: G 't Hooft et al., eds., Recent Developments in Field Theories (Plenum Press, New York, 1980);

E. Witten, Nucl.Phys. B188 (1981) 513;

R.K. Kaul, Phys.Lett. 109B (1982) 19. 
[20] For a review, see: E. Farhi and L. Susskind, Phys.Rep. 74C (1981) 277;

S. Dimopoulos and L. Susskind, Nucl.Phys. B155 (1979) 237;

E. Eichten and K. Lane, Phys.Lett. B90 (1980) 125;

M.E. Peskin and T. Takeuchi, Phys.Rev. D46 (1992) 381;

G. Altarelli, R. Barbieri and S. Jadach, Nucl.Phys. B369 (1992) 3.

[21] P. Fayet and S. Ferrara, Phys.Rep. 32 (1977) 251;

H.E. Haber and G.L. Kane, Phys.Rep. 117 (1985) 75.

[22] See, e.g., C. Kounnas, A.B. Lahanas, D.V. Nanopoulos and M. Quirós, Phys.Lett. 132B (1983) 95.

[23] M. Grünewald and D. Karlen, talks at International Conference on High-Energy Physics, Vancouver 1998,

http: //wWw . cern. ch/LEPEWWG/misc.

[24] Y. Okada, M. Yamaguchi and T. Yanagida, Progr.Theor.Phys. 85 (1991) 1;

J. Ellis, G. Ridolfi and F. Zwirner, Phys.Lett. B257 (1991) 83, Phys.Lett. B262 (1991) 477 ;

H.E. Haber and R. Hempfling, Phys.Rev.Lett. 66 (1991) 1815;

R. Barbieri, M. Frigeni and F. Caravaglios, Phys.Lett. B258 (1991) 167;

Y. Okada, M. Yamaguchi and T. Yanagida, Phys.Lett. B262 (1991) 54.

[25] J. Ellis, S. Kelley and D.V. Nanopoulos, Phys.Lett. B249 (1990) 441 and Phys.Lett. B260 (1991) 131;

U. Amaldi, W. de Boer and H. Furstenau, Phys.Lett. B260 (1991) 447;

P. Langacker and M. Luo, Phys.Rev. D44 (1991) 817.

[26] J. Ellis, J.S. Hagelin, D.V. Nanopoulos, K.A. Olive and M. Srednicki, Nucl.Phys. B238 (1984) 453.

[27] J. Rich, M. Spiro and J. Lloyd-Owen, Phys.Rep. 151 (1987) 239;

P.F. Smith, Contemp.Phys. 29 (1998) 159;

T.K. Hemmick et al., Phys.Rev. D41 (1990) 2074.

[28] R.N. Mohapatra and S. Nussinov, Phys.Rev. D57 (1998) 1940.

[29] J. Ellis, T. Falk, K. Olive and M. Schmitt, Phys.Lett. B388 (1996) 97 and Phys.Lett. B413 (1997) 355.

[30] H.V. Klapdor-Kleingrothaus and Y. Ramachers, Eur.Phys.J. A3 (1998) 85.

[31] G. Giudice and R. Rattazzi, hep-ph/9801271.

[32] J. Ellis, T. Falk, K. Olive and M. Schmitt, Phys.Lett. B413 (1997) 355. 
[33] LEP Experiments Committee meeting, Nov. 12th, 1998, http:www . cern. ch/Committees/LEPC/minutes/LEPC50.html.

This source provides some preliminary updates of this and other experimental plots from papers contributed to the International Conference on High-Energy Physics, Vancouver 1998:

http://ichep.triumf.ca/main.asp.

[34] J. Ellis, K. Enqvist, D.V. Nanopoulos and F. Zwirner, Mod.Phys.Lett. A1 (1986) 57;

G.F. Giudice and R. Barbieri, Nucl.Phys. B306 (1988) 63.

[35] P.H. Chankowski, J. Ellis and S. Pokorski, Phys.Lett. B423 (1998) 327;

P.H. Chankowski, J. Ellis, M. Olechowski and S. Pokorski, hep-ph/9808275.

[36] P.H. Chankowski, J. Ellis, K.A. Olive and S. Pokorski, hep-ph/9811284.

[37] K.A. Olive and M. Srednicki, Phys.Lett. B230 (1989) 78 and Nucl.Phys. B355 (1991) 208; K. Griest, M. Kamionkowski and M.S. Turner, Phys.Rev. D41 (1990) 3565.

[38] J. Ellis, T. Falk and K.A. Olive, hep-ph/9810360.

[39] S. Abel and T. Falk, hep-ph/9810297.

[40] S. Abdullin and F. Charles, hep-ph/9811402.

[41] E.W. Kolb, D.J.H. Chung and A. Riotto, hep-ph/9810361 and references therein.

[42] K. Greisen, Phys.Rev.Lett. 16 (1966) 748;

G.T. Zatsepin and V.A. Kuzmin, Pis'ma Zh.Eksp.Teor.Fiz. 4 (1966) 114.

[43] A.A. Watson, talk at this meeting;

M. Takeda et al., Phys.Rev.Lett. 81 (1998) 1163 and references therein.

[44] V. Berezinsky and A. Mikhailov, astro-ph/9810277;

G.A. Medina Tanco and A.A. Watson, Dark Matter Halos and the Anisotropy of Ultra-High Energy Cosmic Rays, November 1998.

[45] K. Benakli, J. Ellis and D.V. Nanopoulos, hep-ph/9803333.

[46] J. Ellis, J. Lopez and D.V. Nanopoulos, Phys.Lett. B247 (1990) 257;

J. Ellis, G. Gelmini, J. Lopez, D.V. Nanopoulos and S. Sarkar, Nucl.Phys. B373 (1992) 399.

[47] V. Berezinsky, M. Kachelriess and A. Vilenkin, Phys.Rev.Lett. 79 (1997) 4302;

V. Berezinsky and M. Kachelriess, Phys.Lett. B434 (1998) 61.

[48] M. Birkel and S. Sarkar, Astropart.Phys. 9 (1998) 297.

[49] N. Bahcall, talk at this meeting; see also N.A. Bahcall and X.H. Fan, astro-ph/9804082. 
[50] A.G. Riess et al., astro-ph/9805201;

S. Perlmutter et al.,astro-ph/9812133.

[51] P.J. Steinhardt, talk at this meeting; see also

I. Zlatev, L.M. Wang, P.J. Steinhardt, astro-ph/9807002.

[52] J. Ellis, N.E. Mavromatos and D.V. Nanopoulos, gr-qc/9810086. 